\title{
Regard sur un don exceptionnel à la Médiathèque du musée du quai Branly- Jacques-Chirac
}

\author{
Sarah Frioux-Salgas
}

\begin{abstract}
Citer cet article: Frioux-Salgas Sarah (2021), « Regard sur un don exceptionnel à la Médiathèque du musée du quai Branly-Jacques-Chirac », Revue d'Histoire Contemporaine de l'Afrique, en ligne. URL:
\end{abstract}

https://oap.unige.ch/journals/rhca/article/view/stcfrioux

Mise en ligne : 7 janvier 2021

DOI : https://doi.org/10.51185/journals/rhca.2021.e288

Sarah Frioux-Salgas, responsable des archives au musée du Quai Branly-Jacques Chirac, inaugure la rubrique Sources, terrains et contextes de la RHCA en présentant I'histoire et la teneur du don réalisé juste avant sa mort en 2018 par Michel de Breteuil à la médiathèque et aux archives du musée. Michel de Breteuil, fils du magnat de la presse coloniale Charles de Breteuil, fut ancien directeur de la revue Bingo et fondateur du magazine Amina. Il a ainsi rendu accessible les collections complètes de dix revues ouest-africaines parues au cours du 20 ème siècle et d'environ 20000 photographies et négatifs qui en constituait la base iconographique. Si les premières sont déjà consultables à la médiathèque du musée, les secondes font en ce moment l'objet d'un immense chantier de reconditionnement et d'inventaire avant leur mise à disposition des chercheurs.

\section{Médiathèque du quai Branly : ressources pour une histoire culturelle du continent africain}

En 2006, lorsque le musée du quai Branly-Jacques Chirac ouvre ses portes, les enjeux concernent essentiellement la nouvelle présentation d'œuvres originaires d'Afrique, du Proche-Orient, d'Asie, d'Océanie et des Amériques présentes dans les collections publiques françaises depuis quatre siècles, la mise en ligne de leur inventaire, mais aussi la proposition d'un nouvel appareil critique sur le plateau des collections ${ }^{1}$. Les conditions de création du musée mais aussi les choix scientifiques et muséographiques donnent alors lieu à de nombreux débats. Le musée est aussi un lieu dédié à la recherche et il abrite un département de la recherche et de l'enseignement ainsi qu'une importante médiathèque, héritière des collections du musée de l'Homme et du musée des arts d'Afrique et d'Océanie (MNAO)², constituée d'une

\footnotetext{
${ }^{1}$ Le Musée du quai Branly-Jacques Chirac est l'héritier de deux institutions : le musée de l'Homme et le musée des arts d'Afrique et d'Océanie. URL : http://www.quaibranly.fr/fr/collections/toutes-les-collections/histoire-des-collections/ (consulté le 14 décembre 2020). L'exposition 20 ans : les acquisitions du musée du quai Branly - Jacques Chirac (24 septembre 2019 au 26 janvier 2020) est largement revenue sur l'histoire des collections du musée. Voir aussi Delpuech André, Laurière Christine, Peltier-Caroff Carine (2017), Les années folles de l'ethnographie : Trocadéro 28-37, Paris, Muséum national d'histoire naturelle.

${ }^{2}$ Le musée national des arts d'Afrique et d'Océanie était lui-même l'héritier de l'évolution de cette institution du musée de la France d'outre-mer (1931-1960) et le musée de l'Homme du musée d'ethnographie du Trocadéro (1878-1938).
} 
iconothèque, d'une bibliothèque et d'un service d'archives. Le musée du quai Branly-Jacques Chirac est rarement associé à la richesse des fonds qui y sont conservés, dont certains sont fondamentaux pour étudier la période coloniale mais aussi l'histoire culturelle du continent africain et de sa diaspora. Nous avons notamment pu constater la valeur des collections de périodiques à partir de 2009, lorsque le musée a entamé une série d'expositions consacrées à I'histoire de la négritude et du panafricanisme3. Nous avons par exemple découvert que, dès les années 1930, les bibliothécaires du musée d'Ethnographie du Trocadéro avaient acquis la célèbre revue des sœurs Nardal, La revue du monde noir (1931-1932), ainsi que des numéros de la fameuse revue africaine-américaine The Crisis, fondée en 1910 par le sociologue W.E.B Dubois. Plus tard celles du musée de l'Homme s'étaient abonnées à la revue camerounaise Abbia (1962-1983) ou encore à Liaison (1950-1960, revue du cercle culturel de l'Afrique Équatoriale Française - AEF). La valorisation des périodiques de la médiathèque prit surtout de l'ampleur lorsque Zahia Rahmani (écrivain et responsable du domaine Histoire de l'art mondialisée à I'Institut national d'histoire de l'art - INHA) nous proposa de collaborer à un programme de recherche de I'INHA, "Art Global et Périodiques Culturels. Généalogies critiques et artistiques, XIXe-XXe siècles ». Cette collaboration fut donc pour nous l'occasion de réaliser un important travail de prospection et de valorisation de nos collections de périodiques produits en contexte colonial et postcolonial ${ }^{4}$. Nous avons aussi continué d'enrichir nos fonds en achetant par exemple l'unique numéro de la revue Légitime Défense élaborée à Paris en 1932 par de jeunes étudiants martiniquais communistes et proches des surréalistes mais aussi la collection complète de Black Orpheus. A Journal of African and AfroAmerican Literature lancé en 1957 à Ibadan au Nigéria, par deux expatriés allemands, Ulli Beier et Janheinz Jahn. Lors de l'exposition 20 ans : les acquisitions du musée du quai Branly-Jacques Chirac, nous avons réussi à mettre en avant ces nouvelles acquisitions, montrant ainsi que notre institution ne s'intéressait plus uniquement aux œuvres africaines mais aussi à l'histoire culturelle du continent.

\section{De Panafest Archives à Bingo}

En 2008, c'est lors de la préparation de l'exposition Présence Africaine. Une tribune, un mouvement, un réseau, présentée au musée du quai Branly en 2009, que nous avons constaté la présence à la bibliothèque et aux archives de nombreux documents concernant le Premier Festival mondial des Arts nègres de Dakar (avril 1966), et notamment le fameux numéro de Bingo qui lui fut consacré. En effet, Pierre Meauzé, conservateur au MNAO, et Michel Leiris et Jacqueline Delange, responsables des collections africaines au musée de l'Homme, avaient été impliqués dans l'organisation de l'exposition d'art ancien présentée au musée Dynamique de

\footnotetext{
3 Les quatre autres expositions sont: Présence Africaine. Une tribune, un mouvement, un réseau (2009), L'atlantique noir de Nancy Cunard. Negro Anthology (1931-1934) (2014), Dakar 66, Chronique d'un festival panafricain (2016) et Paul Robeson. Un homme du "Tout Monde » (2018).

4 URL : https://www.inha.fr/fr/recherche/le-departement-des-etudes-et-de-la-recherche/domaines-de-recherche/histoire-de-l-artmondialisee/art-global-et-periodiques-culturels.html (consulté le 14 décembre 2020). Le recensement des revues identifiées dans le cadre de ce vaste projet est aujourd'hui accessible sur le site de I'INHA et donnera bientôt lieu à un portail permettant de localiser ou de consulter ces dernières. URL : https://agorha.inha.fr/inhaprod/ark:/54721/00171 (consulté le 14 décembre 2020). À partir de ce travail, Zahia Rhamani a réalisé la magnifique installation Sismographie des luttes. Vers une histoire globale des revues critiques et culturel/es. En ligne. URL : https://www.inha.fr/fr/agenda/parcourir-par-annee/en-2017/novembre-2017/sismographie-des-luttes-versune-histoire-globale-des-revues-critiques-et-culturelles.html (consulté le 14 décembre 2020).
} 
Dakar ${ }^{5}$. Nous avions donc dans nos archives de nombreux documents institutionnels mais aussi non institutionnels liés à cet évènement, produits ou collectés par ces derniers ${ }^{6}$. Présenté dans la dernière section de l'exposition Présence Africaine, consacrée au festival, ce numéro de Bingo fut de nouveau exposé, en 2016, dans l'exposition Dakar 66. Chroniques d'un festival panafricain, organisée avec les chercheurs Dominique Malaquais et Cédric Vincent, fondateurs et animateurs du projet de recherche collaboratif Panafest Archive. Ce dernier a pour thématique centrale quatre grands festivals panafricains : le Premier Festival mondial des Arts nègres (Dakar, 1966), le Premier Festival culturel panafricain (Alger, 1969), le Festival Zaïre 74 (Kinshasa, 1974) et le Second Festival mondial des Arts nègres (Lagos, 1977). Il a donné lieu à la collecte de documents pour la plupart non institutionnels produits pour et sur les festivals (brochures, ouvrages, affiches, photographies, tracts publicitaires, catalogues d'exposition, disques, journaux, flyers, etc.) et à la réalisation de nombreux entretiens audiovisuels? ${ }^{7}$. Entre l'exposition et la décision de Dominique Malaquais et Cédric Vincent de donner aux archives du musée l'extraordinaire ensemble constitué pour Panafest Archive, nous avons continué à travailler ensemble pour enrichir ce fonds. C'est dans ce contexte que nous avons eu l'occasion de rencontrer l'ancien directeur de Bingo, Michel de Breteuil (1927-2018), fils du magnat de la presse coloniale Charles de Breteuil, et que celui-ci a décidé de faire un don important au musée du quai Branly-Jacques Chirac.

Le mensuel Bingo suscite en outre l'intérêt renouvelé de chercheurs issus de disciplines diverses, permettant de mettre en valeur son caractère panafricain. Dès 2012, I'historienne de la photographie Jennifer Bajorek centre son article " "Ça Bouscoulait!": Democratization \& Photography in Senegal » sur la place de la photographie dans Bingo ${ }^{8}$. En 2018, c'est dans le cadre du colloque organisé par les sociologues Claire Ducournau et Ruth Bush à l'université de Montpellier « Presse et littérature africaine(s) », que l'importance d'un périodique populaire tel que Bingo est interrogée. Les deux chercheuses montrent que « depuis les bulletins coloniaux jusqu'aux revues en ligne, en passant par les magazines illustrés, le périodique a constitué, pour les écrivains issus d'Afrique, un support de publication décisif à côté du livre ${ }^{9}$ ». La même année, c'est au tour de la grande revue d'art africain contemporain NKA ${ }^{10}$ de consacrer un article à Bingo: « Bingo Magazine in the Age of Pan-African Festivals: A Feminist Archive of Global Black Consciousness » dans son numéro dédié à la « Global Black Consciousness ${ }^{11}$ ». Puis, c'est dans

\footnotetext{
${ }^{5}$ Exposition « Art nègre : Sources, Évolutions, Expansion ».

${ }^{6}$ Frioux-Salgas Sarah (2020), "Le $1^{\text {er }}$ festival des arts nègres au sein des collections du musée du quai Branly-Jacques Chirac », in I. Wane et S. Mbaye (dir.), Le 1er Festival mondial des Arts nègres. Mémoire et actualité, Dakar, L'Harmattan-Sénégal, pp. $255-267$.

${ }^{7}$ Voir URL : http://webdocs-sciences-sociales.science/panafest/\#00-Menu-Inroduction_PANAFEST__une_archive_en_devenir (consulté le 14 décembre 2020).

${ }^{8}$ Article publié dans l'ouvrage coordonné par Vocke Richard (2012) (dir.), Photography in Africa: Ethnographic Perspectives, Woodbridge and New York, James Currey. Elle est aussi I'auteure de (2020) Unfixed Photography and Decolonial Imagination in West Africa, Duke, Duke University Press.

${ }^{9}$ Voir URL : http://theatre.univ-montp3.fr/spectacle/2017-2018/colloque-presse-et-litterature-africaines et https://www.fabula.org/actualites/presse-et-litterature-africaines_83848.php (consulté le 14 décembre 2020)

${ }^{10}$ Nka: Journal of Contemporary African Art est fondé en 1994 à New York par Okwui Enwezor qui introduit sa préface par ces lignes : "Lorsqu'on visite les musées, les galeries d'art ou tout lieu de valorisation culturelle en Europe et aux États-Unis, I'un des aspects problématiques est l'absence totale, dans ces environnements hautement surveillés, d'art réalisé par des artistes contemporains africains. Non seulement les œuvres de ces artistes (dont beaucoup travaillent depuis cinquante ans) sont absentes du musée et de la galerie, mais on leur a accordé tout aussi peu d'attention dans les pratiques académiques de l'histoire de l'art que dans les programmes d'études universitaires, les médias ou tout autre organe de presse ».

11 Jaji Tsitsi (2018), "Bingo Magazine in the Age of Pan-African Festivals: A Feminist Archive of Global Black Consciousness », NKA, Journal of Contemporary African Art, 42-43, pp. 110-123.
} 
la continuité de leur colloque de 2018 que Claire Ducournau et Ruth Bush coordonnent au printemps 2020 un numéro de la revue Research in African Literatures : «African Audiences: Making Meaning across Media12 ${ }^{12}$.

\section{L'histoire de Bingo}

Lorsqu'en 1953, Ousmane Socé sollicite la famille de Breteuil pour fonder un journal, il termine à peine sa courte carrière politique (1946-1952) au sein de la $\mathrm{SFIO}^{13}$. Né à Rufisque en 1911, il est passé par l'École normale William Ponty de Gorée avant d'obtenir une bourse pour faire des études de vétérinaire en France dans les années 1930. II est aussi l'auteur de plusieurs textes et poèmes publiés en métropole: Karim. Roman sénégalais (1935), Mirages de Paris (1937), Contes et légendes d'Afrique noire (1938) et Rythmes du Khalam (1948) ${ }^{14}$. La création de la revue Bingo est donc le fruit de l'association d'un intellectuel-homme politique sénégalais soucieux de s'adresser à tous les publics et du fils de Charles de Breteuil, I'homme de presse le plus puissant d'Afrique de l'Ouest qui avait notamment fondé en 1935 Paris-Dakar, puis Abidjan Matin en 1952.

Cette alliance entre de jeunes intellectuels sénégalais et la famille de Breteuil n'était pas inédite. En janvier 1942, Charles de Breteuil avait créé Dakar-Jeunes, un supplément du quotidien Paris-Dakar, auquel avait participé Ousmane Socé ${ }^{15}$. Ce dernier y avait publié dans les quatre premiers numéros, sous le nom de Karamoko, une série d'articles intitulés ${ }^{16}$ : "Vous de France, nous d'Afrique » et, sous son vrai nom : "Un témoignage : l'évolution culturelle de I'AOF ». Socé n'est donc pas un novice lorsqu'il sollicite la famille de Breteuil. En tant qu'écrivain, journaliste ou homme politique, il garde le souci de s'adresser à un large public. Dans son article publié récemment, Tobias Warner propose une analyse très intéressante du projet de ce dernier qui s'inscrit parfaitement dans son parcours intellectuel et politique. Warner montre clairement la place que Socé souhaitait donner aux lecteurs dans son journal, les interpellant ainsi dans le premier numéro : "Écrivez-nous pour nous dire ce que vous pensez, ce que vous désirez trouver... participez à la vie du journal en envoyant des photos ${ }^{17}$ ». Selon Michel de Breteuil, ce périodique se voulait une version francophone des magazines

\footnotetext{
12 Les deux chercheuses avaient aussi organisé en 2017 à la Maison de la femme-Henriette Bathily (Dakar) la très belle exposition «Awa, une revue féminine pionnière. Sénégal 1963-1974 » et surtout avaient permis la mise en ligne de cette revue fondée par la grande journaliste Annette Mbaye. Voir URL https://www.awamagazine.org/fr/qui-sommes-nous/ (consulté le 14 décembre 2020).

${ }^{13}$ Warner Tobias (2020), « On the Hospitality of Print: Ousmane Socé's Bingo and Its Publics », Research in African Literatures, 51(1), pp. 21-44.

${ }^{14}$ Concernant les premiers écrivains africains de langue française, voir les textes de l'exposition organisée aux Archives nationales d'outre-mer (ANOM) à Aix-en-Provence à l'occasion du Congrès 2019 de l'Association pour l'étude des littératures africaines (APELA) : Archives matérielles, traces mémorielles \& littératures des Afriques, 25-27 septembre 2019. Exposition conçue et réalisée par Elara Bertho, Catherine Mazauric, Cécile van den Avenne, avec le soutien d'Isabelle Dion, Frédéric Gilly, Sylvie Pontillo des ANOM. URL : https://hal.archives-ouvertes.fr/hal-02291167/document et le carnet de recherche l'accompagnant https://elam.hypotheses.org/2216 (consulté le 14 décembre 2020).

${ }^{15}$ À propos des élites africaines des années 1930-1940, voir Jézéquel Jean-Hervé (2005), "Les enseignants comme élite politique en AOF (1930-1945) », Cahiers d'études africaines, 178, pp. 519-543. Voir aussi du même auteur : (2012), "La vie culturelle dans les villes de l'empire colonial français. "Nous n'avons pas à maquiller l'Afrique" Jeunesse lettrée et débats sur la culture dans le Dakar vichyste ", in Fr. Talioano-des-Garets (dir.), Paris, Armand Colin, pp. 88-97. Voir aussi Labrune-Badiane Céline et Smith Etienne (2018), Les Hussards noirs de la colonie. Instituteurs et petites patries en AOF (1913-1960), Paris, Khartala et le carnet hypothèses du projet. URL : https://bibcolaf.hypotheses.org/1686 (consulté le 14 décembre 2020).

${ }^{16}$ Dakar-Jeunes, $\mathrm{n}^{\circ} 1,8$ janvier $1942 ; \mathrm{n}^{\circ} 2,15$ janvier $1942 ; \mathrm{n}^{\circ}$ 3, 22 janvier $1942 ; \mathrm{n}^{\circ}$ 4, 29 janvier 1942

${ }^{17}$ Warner T., « On the Hospitality... », art. cité., p. 30
} 
populaires et très illustrés Drum (créé en 1951 en Afrique du Sud) et Ebony-destiné au public africain-américain - fondé en 1945 à Chicago.

Tirés dans les années 1960 à environ 8000 exemplaires, Bingo a de nombreux abonnés et une très grande audience en Afrique francophone, essentiellement au Sénégal, mais aussi en Côte d'Ivoire ou au Cameroun. La popularité et la diversité de son lectorat sont certainement dues aux sujets des articles. On y trouve pêle-mêle des romans-photos, des conseils de coiffure, des textes littéraires, un courrier du cœur, des critiques de films, des récits historiques ou encore des portraits d'intellectuels, d'artistes ou de sportifs : Édouard Glissant, Miriam Makeba, Patrice Lumumba, Malcom X ou le footballeur Pelé.

\section{Le don d'un fonds exceptionnel}

En 2017, lorsque nous prenons contact avec lui, Michel De Breteuil a 90 ans et est toujours le rédacteur en chef du magazine féminin Amina qu'il a fondé en $1972^{18}$. Souhaitant que Bingo puisse être accessible facilement et à tous, il propose dans un premier temps de donner une collection complète à la médiathèque du musée du Quai Branly. C'est en commençant I'organisation pratique de ce don que Michel de Breteuil nous propose - à mon collègue PierreYves Belfils, responsable des périodiques et à moi-même - de nous rendre dans une maison située en banlieue parisienne dans laquelle il conserve diverses archives qu'il veut nous montrer et certainement nous donner, ne sachant pas qu'en faire. En y arrivant nous découvrons d'abord I'héritage de la famille de Breteuil, c'est-à-dire des piles allant - au sens propre du terme - jusqu'au plafond de numéros reliés de tous les journaux fondés par son père : Abidjan Matin (1954-1964), Dakar Matin (1961-1970), Dakar-jeunes (1942), Guinée matin (1958), La dépêche marocaine de Tanger (1905-1956), La presse de Guinée (1953), La presse du Cameroun (1955-1974), Paris-Dakar (1934-1961) et Paris Tana (1936-1939). Dans un second temps, Michel de Breteuil nous emmène dans une autre pièce où sont conservées des dizaines de boîtes remplies de photographies classées par pays et par thèmes, que les équipes de la rédaction de Bingo utilisaient pour illustrer leurs articles. Cet ensemble était donc la source d'un périodique dans lequel l'image avait une place majeure. Après cette visite et une réflexion sur la possibilité de traiter, conserver et diffuser ces fonds extraordinaires, nous acceptons qu'il complète son don avec les collections quasi-complètes des quotidiens cités et les photographies.

Ces centaines de photographies de presse - légendées ou pas - sont une acquisition très importante. Elles illustrent de manière très variée I'histoire culturelle et politique du continent africain des années 1950 aux années 1990. Le traitement physique, juridique et intellectuel de cet immense fonds photographique sera néanmoins très long (contrairement aux périodiques qui sont disponibles à la bibliothèque depuis deux ans), et nécessitera une collaboration étroite avec des chercheurs quand nous aurons terminé le reconditionnement. II s'agit de la première étape de ce chantier que nous avons commencée au début de cette année, pour traiter ce vaste ensemble constitué d'environ 20000 documents (tirages et négatifs). Cette étape est cruciale, car il est fondamental d'être très vigilant pour ne perdre aucune des informations qui

\footnotetext{
18 Sassoon Virginie (2012), "La représentation du couple dans le magazine féminin Amina: une vision androcentrique », Le Temps des médias, 19, pp. 145-158.
} 
contextualisent ce fonds (thèmes indiqués sur les boîtes, mode de classement initial, etc.), afin de comprendre la manière dont il a été constitué. Ce travail indispensable en amont permettra ainsi de le rendre accessible dans les deux ans à venir et de mettre en place différents projets de recherche, à la fois pour l'étudier et le valoriser. L'autre enjeu scientifique de ce projet sera de mettre ces périodiques et ces archives à la disposition de nos collègues du continent africain qui travaillent sur ces sujets sans avoir accès aux sources. Nous espérons donc pouvoir financer des projets permettant d'imaginer collectivement la forme que devra prendre le partage et la restitution de ces archives sénégalaises et panafricaines conservées en France ${ }^{19}$.

Sarah Frioux-Salgas

Responsable des archives au Musée du Quai Branly-Jacques Chirac (France)

\section{Bibliographie}

BAJOREK Jennifer (2012), « "Ça Bouscoulait!": Democratization \& Photography in Senegal », in R. Vocke (dir.), Photography in Africa: Ethnographic Perspectives, Woodbridge and New York, James Currey, pp. 140-165.

(2020) Unfixed Photography and Decolonial Imagination in West Africa, Durham, Duke University Press.

Delpuech André, Lauriere Christine, Peltier-Caroff Carine (2017), Les années folles de l'ethnographie: Trocadéro 28-37, Paris, Muséum national d'histoire naturelle.

FRIOUX-SALGAS Sarah (2020), "Le $1^{\text {er }}$ festival des arts nègres au sein des collections du musée du quai Branly-Jacques Chirac », in I. WANE et S. MBAYE (dir.), Le 1er Festival mondial des Arts nègres. Mémoire et actualité, Dakar, L'Harmattan-Sénégal, pp. 255-267.

JAJI Tsitsi (2018), « Bingo Magazine in the Age of Pan-African Festivals: A Feminist Archive of Global Black Consciousness », NKA, Journal of Contemporary African Art, 42-43, pp. 110-123.

JeZEQUEL Jean-Hervé (2005), "Les enseignants comme élite politique en AOF (1930-1945) », Cahiers d'études africaines, 178, pp. 519-543.

(2012), « La vie culturelle dans les villes de l'empire colonial français. "Nous n'avons pas à maquiller l'Afrique" Jeunesse lettrée et débats sur la culture dans le Dakar vichyste », in Fr. TALIOANO-DESGARETS (dir.), Paris, Armand Colin, pp. 88-97.

LABRUNe-BADIANE Céline et SMITH Etienne (2018), Les Hussards noirs de la colonie. Instituteurs et petites patries en AOF (1913-1960), Paris, Khartala.

SASSOON Virginie (2012), "La représentation du couple dans le magazine féminin Amina: une vision androcentrique », Le Temps des médias, 19), pp. 145-158.

\footnotetext{
19 La médiathèque du musée du quai Branly-Jacques Chirac a déjà commencé cette démarche dans le cadre du Labex Les passés dans le présent. URL: http://passes-present.eu/fr/pour-un-partage-des-archives-le-festival-mondial-des-arts-negres-dakar-196644319 (consulté le 14 décembre 2020)
} 
WARner Tobias (2020), " On the Hospitality of Print: Ousmane Socé's Bingo and Its Publics », Research in African Literatures, 51(1), pp. 21-44. 